\title{
UTILIZAÇÃO DA DISTRIBUIÇÃO INVERSA DE CHEN PARA A MODELAGEM DOS TEMPOS DE SOBREVIVÊNCIA DE COBAIAS INOCULADAS PELO VÍRUS DA TUBERCULOSE
}

\author{
USE OF THE CHEN INVERSE DISTRIBUTION TO THE MODELING OF THE \\ SURVIVAL TIMES OF GUINEA PIGS INOCULATED BY VIRUS \\ TUBERCULOSIS.
}

Paulo Roberto de Lima Gianfelice, Sérgio Minoru Oikawa, Fernando Antônio Moala Universidade Estadual Paulista - UNESP - Faculdade de Ciências e Tecnologias,
Departamento de Estatística, Presidente Prudente, São Paulo.
E-mail: geanfelice@ @otmail.com

RESUMO - Com o intuito de modelar o tempo em que o curso de uma patologia se desenvolve, o presente trabalho empregou os conceitos de análise de sobrevivência no desenvolvimento de um modelo estatístico para de estudar os tempos de vida dos hospedeiros do vírus da tuberculose. Com base nos resultados, constatou-se que o modelo probabilístico Inversa de Chen forneceu um excelente ajuste aos dados de sobrevivência, quando eles apresentam função de risco unimodal. Além disso, concluiu-se que, numa comparação com outros modelos propostos pela literatura, o modelo em questão se mostrou o mais adequado para analisar e descrever os dados sobre os tempos de vida no desenvolvimento da infecção por tuberculose.

Palavras-chave: Ajustamento de Curva; Análise de Sobrevivência; Análise Estatística; Modelagem Estatística; Modelo de Risco.

ABSTRACT - With the intention to model the time on what the course of an pathology if develops, in this study we used the survival analysis concepts to obtain a statistical model to be used in life span study of host of the virus the tuberculosis. Based on the results, it was found that the probabilistic model Chen Inverso provided an excellent fit to survival data, since these showed unimodal risk curve. Besides that, it was concluded in a comparison with other models proposed in the literature that the model in question if showed to be most suitable to analyze and describe the life-time data in development of infection tuberculosis.

Keywords: Adjustment Curve; Statistical Modeling; Statistical Analysis; Survival Analysis; Risk Model. 


\section{INTRODUÇÃO}

A infecção por bacilo da tuberculose (TB) representa um grave problema de saúde pública, que de acordo com Sant'Anna e Hijjar (2007), só no Brasil, estima-se a ocorrência de 111.000 novos casos hospitalares por ano, com cerca de 6.000 óbitos, indicando a necessidade e importância de estudos em caráter preventivo e de combate desta patologia.

No entanto, uma análise dos resultados de um experimento de inoculação deste vírus realizados em 1414 cobaias animais (porcos da índia) foi interpretada por Bjerkedal (1960) como um mostrador de que as diferenças de virulência dos bacilos infectantes só mudam o aspecto quantitativo da relação entre os números iniciais de bacilos infectantes e o tempo de sobrevivência das cobaias. O estudo, portanto, deixa claro o uso de cobaia animal e a sua dependência direta da análise de sobrevivência em seus respectivos tempo de vida.

Segundo Morales (2008, p. 33-36), ao contrário do que muitos pensam, o interesse por métodos alternativos em experimentação tem crescido na comunidade científica no intuito de reduzir o número de animais utilizados em experimentos. Dessa forma, como método alternativo, Morales destaca em suas propostas que,
[...] os modelos matemáticos podem contribuir para o trabalho experimental através da definição de variáveis e testes de teorias, reduzindo o custo desses experimentos e os tornando mais eficazes. [...] computadores processam e armazenam conhecimentos já existentes e muitos deles foram adquiridos com a utilização de animais na pesquisa.

Nesse contexto, a partir de um conjunto de dados correspondentes aos tempos de sobrevivência (em dias) de cobaias animais, o presente estudo teve como objetivo propor um modelo estatístico com a finalidade de analisar os tempos de vida dos porcos da índia infectados com o bacilo da tuberculose. E em particular, associar-se às técnicas de análise de sobrevivência como um método alternativo no refinamento dos modelos matemáticos tomados para simular a utilização de cobaia animal em pesquisas científicas.

\section{METODOLOGIA}

\section{Análise de Sobrevivência}

A análise de sobrevivência é a metodologia adotada neste trabalho, e é definida como um método estatístico usado para análise de dados de tempo de vida, comumente derivados de estudos de laboratórios ou de clínicas relacionadas à Biomedicina em tratamentos de doenças agudas, severas ou fatais, e em áreas de conhecimento em geral.

Geralmente, estuda-se o tempo em que uma unidade experimental sobrevive a um 
determinado tratamento, isto é, o tempo de resposta a um dado evento ou o tempo em que uma unidade experimental desenvolveu uma dada doença específica.

Comumente, no tratamento dos dados efetuam-se as estatísticas descritivas e gráficas e em paralelo realizam-se inferências e testes das estimativas levantadas sobre os dados de sobrevivência pelos métodos do EMV (estimador de máxima verossimilhança) e o EKM (estimador de Kaplan - Meyer) a fim de tomar os resultados obtidos à cerca da experimentação de interesse para se alcançar as conclusões mais precisas e confiáveis sobre o caso praticado. Para maiores detalhes, sugere-se consultar Colosimo e Giolo (2006).

O Modelo Proposto: A Distribuição Inversa de Chen com Dois Parâmetros Inicialmente, citado por Srivastava e Srivastava (2014), Chen (2000) propôs uma distribuição de probabilidade com dois parâmetros a fim de estudar os tempos de vida, denominada distribuição de Chen. Se $X$ é uma variável aleatória com função densidade de probabilidade dada por:

$$
f(x \mid \alpha ; \beta)=\alpha \beta x^{\beta-1} \exp \left\{x^{\beta}+\alpha\left[1-\exp \left(x^{\beta}\right)\right]\right\}
$$

Então, $X$ segue uma distribuição de Chen com parâmetros $\alpha$ e $\beta$. Denota-se $X \sim$ Chen $(\alpha ; \beta)$.

Agora, considere $T$ como sendo uma variável aleatória tal que $\mathrm{T}=\mathrm{X}^{-1}$. Então, utilizando-se o método de transformação de variáveis aleatórias, neste caso, a função de

$$
\begin{gathered}
F(t \mid \alpha ; \beta)=\exp \left\{\alpha\left[1-\exp \left(\mathrm{y}^{-\beta}\right)\right]\right\} \\
f(t \mid \alpha ; \beta)=F^{\prime}(y \mid \alpha ; \beta)=\alpha \beta y^{-(\beta+1)} \exp \left\{y^{-\beta}+\alpha\left[1-\exp \left(y^{-\beta}\right)\right]\right\}
\end{gathered}
$$

em que $\alpha>0$ e $\beta>0$. Dessa forma, $t$ segue uma distribuição inversa de Chen e é denotada por $\mathrm{T} \sim \operatorname{Chen}^{-1}(\alpha ; \beta)$. 


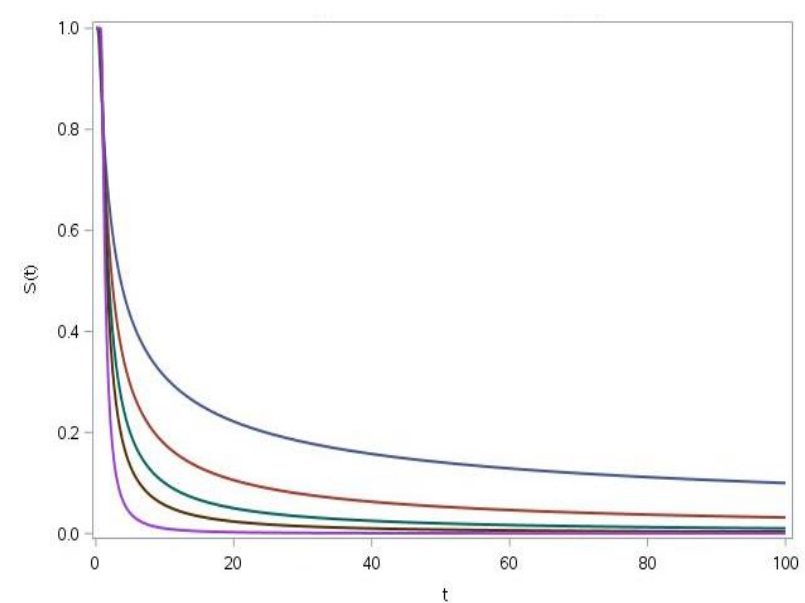

Figura 1. Gráficos ilustrativos de $\mathrm{S}(\mathrm{t})$ para $\mathrm{Chen}^{-1}$

A sua função de sobrevivência, denotada por $S(t)$ é definida como $S(t)=P(T \geq t)$, isto é, a probabilidade de ocorrência do evento $T$ no instante $t$, em que $S(t)=1-$
$F(t)$. Assim, a função de sobrevivência e a função de risco (taxa de falha) são dadas, respectivamente, pelas expressões (4) e (5).

$$
\begin{gathered}
S(t \mid \alpha ; \beta)=1-\exp \left\{\alpha\left[1-\exp \left(\mathrm{t}^{-\beta}\right)\right]\right\} \\
\lambda(t \mid \alpha ; \beta)=\frac{\alpha \beta t^{-(\beta+1)} \exp \left\{t^{-\beta}+\alpha\left[1-\exp \left(\mathrm{t}^{-\beta}\right)\right]\right\}}{1-\exp \left\{\alpha\left[1-\exp \left(\mathrm{t}^{-\beta}\right)\right]\right\}}
\end{gathered}
$$

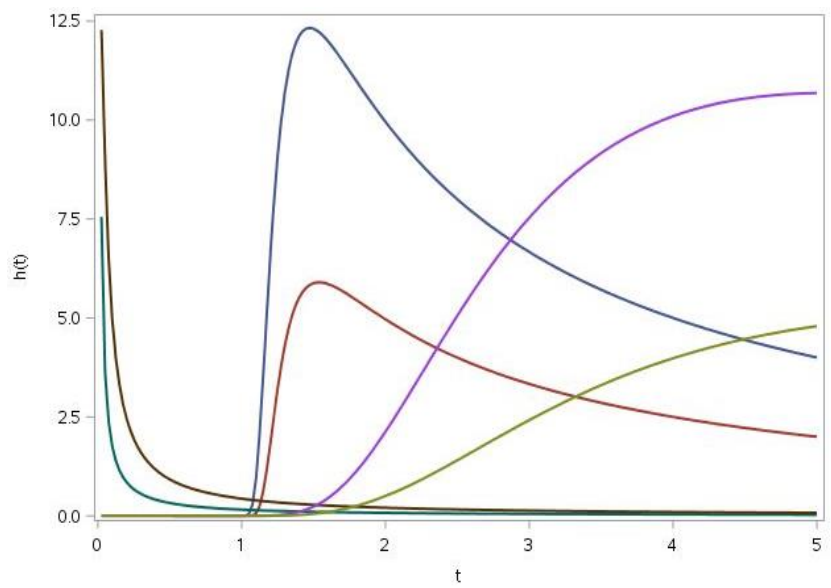

Figura 2. Gráficos ilustrativos de $\lambda(\mathrm{t})$ para $\mathrm{Chen}^{-1}$

A Figura 1 ilustra a função de qualquer valor de $\alpha$ e $\beta$, observa-se que a sobrevivência para diferentes valores dos distribuição de inversa de Chen apresenta parâmetros. De acordo com a Figura 2, para funções de risco, $\lambda(t)$, unimodal, monótona 
decrescente e monótona crescente para alguns intervalos de tempo $t \in\left[t_{1} ; t_{2}\right]$. Neste caso, este modelo é predominantemente côncavo, significando que a distribuição inversa de Chen é adequada para analisar dados com taxa de falha unimodal. Mais detalhes sobre o modelo probabilístico de Chen $^{-1}(\alpha ; \beta)$ pode ser obtido em Srivastava e Srivastava (2014).

\section{O Gráfico do Tempo Total em Teste (TTT)}

Existem diversos procedimentos preliminares para identificar as formas da função de risco em análise de sobrevivência. Dentre eles, destaca-se o método gráfico conhecido como tempo total em teste (TTT) que é comumente empregado em contextos onde existem informações qualitativas sobre a curva de risco.

$$
L(\alpha, \beta \mid x)=\prod_{i=1}^{n} \alpha \beta x_{i}^{-(\beta+1)} \exp \left\{x_{i}^{-\beta}+\alpha\left[1-\exp \left(\mathrm{x}_{\mathrm{i}}^{-\beta}\right)\right]\right\}
$$

O logaritmo da verossimilhança é dado por:

$l(\alpha, \beta \mid x)=n\left(\log (\alpha \beta)-(\beta+1) \sum_{i=1}^{n} \log \left(x_{i}\right)+\sum_{i=1}^{n}\left\{x_{i}^{-\beta}+\alpha\left[1-\exp \left(\mathrm{x}_{\mathrm{i}}^{-\beta}\right)\right]\right.\right.$

Os estimadores de máxima verossimilhança são obtidos como solução das equações dadas por:

$$
\begin{gathered}
\frac{\partial l(\alpha, \beta \mid x)}{\partial \alpha}=\frac{n}{\alpha}+n-\sum_{i=1}^{n} \exp \left(\mathrm{x}_{\mathrm{i}}^{-\beta}\right)=0 \\
\frac{\partial l(\alpha, \beta \mid x)}{\partial \beta}=\frac{n}{\beta}-\sum_{i=1}^{n} \log \left(x_{i}\right)-\sum_{i=1}^{n}\left(x_{i}^{-\beta}\right)+\alpha \sum_{i=1}^{n}\left[\exp \left(\mathrm{x}^{-\beta}\right) x_{i}^{-\beta} \log \left(x_{i}\right)\right]=0
\end{gathered}
$$

Como não existe uma solução fechada para (8) e (9), foram usadas métodos numéricos para encontrar as estimativas de máxima verossimilhança de $\alpha$ e $\beta$. Neste trabalho, utilizaram-se diversos pacotes do 
software R, Livre (www.r-project.org), dentre eles, $\max \operatorname{Lik}()$.

\section{O Conjunto de Dados}

No presente trabalho, a metodologia proposta foi utilizada para analisar o conjunto de dados reais correspondentes aos tempos de sobrevivência, em dias, de 72 cobaias infectadas com doses do bacilo da tuberculose. Tais dados foram extraídos de Bjerkedal (1960) e podem ser visualizados no quadro a seguir.

Quadro 1. Tempo de sobrevivência das cobaias infectadas

\begin{tabular}{|cccccccccccccccccc|}
\hline 12 & 15 & 22 & 24 & 24 & 32 & 32 & 33 & 34 & 38 & 38 & 43 & 44 & 48 & 52 & 53 & 54 & 54 \\
54 & 56 & 57 & 58 & 58 & 59 & 60 & 60 & 60 & 60 & 61 & 62 & 63 & 65 & 65 & 67 & 68 & 70 \\
70 & 72 & 73 & 75 & 76 & 76 & 81 & 83 & 84 & 85 & 87 & 91 & 95 & 96 & 98 & 99 & 109 & 110 \\
121 & 127 & 129 & 131 & 143 & 146 & 146 & 175 & 175 & 211 & 233 & 258 & 258 & 263 & 297 & 341 & 341 & 376 \\
\hline
\end{tabular}

Fonte: Bjerkedal (1960).

\section{RESULTADOS}

Inicialmente,

verificou-se

0

comportamento da função de risco resultante dos dados de sobrevivência do Quadro 1, através do gráfico TTT (Figura 3). Constata-se que o mesmo apresenta a forma unimodal para a função taxa de risco.

Neste caso, assumiu-se a distribuição de inversa de Chen pelo fato dos dados de sobrevivência interceptar o eixo anti-diagonal do gráfico TTT na forma de $S$ invertido, sendo uma parte da curva côncava seguida de outra parte convexa, necessariamente, nesta ordem ao longo do eixo das abscissas. $\mathrm{Na}$ sequência, a fim de realizar comparações dos ajustes da distribuição de $\mathrm{Chen}^{-1}$ com outras distribuições comumente usadas na análise de sobrevivência, consideraram-se as distribuições Log-Normal e Log-Logística que também apresentam risco unimodal. Para tanto, utilizou-se o método de máxima verossimilhança na obtenção dos valores estimados dos parâmetros de forma e escala dos modelos considerados. Os resultados são apresentados no Quadro 1.

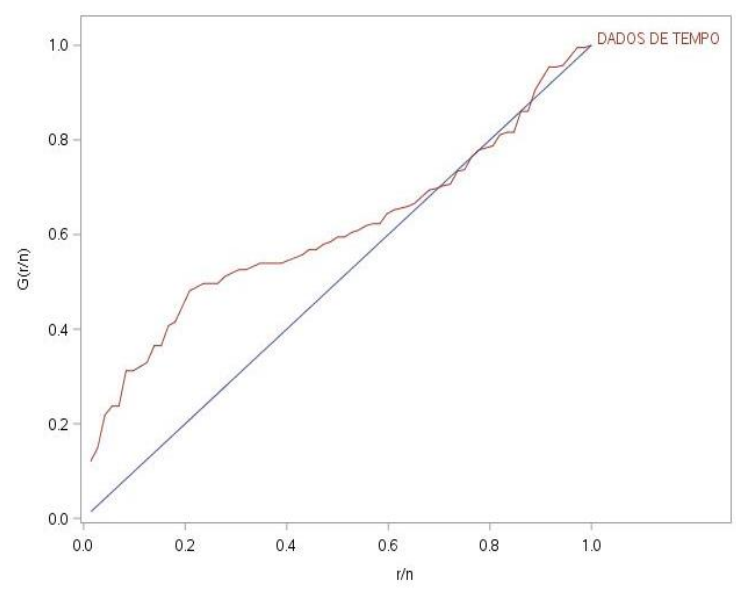

Figura 3. Gráfico TTT para os dados reais 
Com base nestas estimativas, foi possível modelar os comportamentos das funções de sobrevivência e de taxa de risco dos modelos
Log-Normal, log-Logística e Chen $^{-1}$ a fim de compará-los analiticamente e graficamente.

Tabela 1. Estimativas dos parâmetros de forma e escala e os respectivos IC(95\%)

\begin{tabular}{|c|c|c|c|}
\hline Modelo & $(\widehat{\boldsymbol{\alpha}} ; \widehat{\boldsymbol{\beta}})$ & Erros padrões de $(\widehat{\boldsymbol{\alpha}} ; \widehat{\boldsymbol{\beta}})$ & Intervalo 95\% de Confiança \\
\hline CHEN $^{-1}$ & $(282,971 ; 1,415)$ & $(4,224 ; 0,031)$ & $(274,67 ; 291,25) \mathrm{e}(1,35 ; 1,48)$ \\
\hline LOG-NORMAL & $(4,344 ; 1,005)$ & $(0,119 ; 0,118)$ & $(4,11 ; 4,58) \mathrm{e}(0,77 ; 1,24)$ \\
\hline LOG-LOGISTICA & $(75,221 ; 2,482)$ & $(2,966 ; 0,248)$ & $(69,41 ; 81,03) \mathrm{e}(1,99 ; 2,96)$ \\
\hline
\end{tabular}

Analiticamente, a comparação entre os três modelos considerados foram realizados através das respectivas funções penalizadoras, denominadas de critérios de informação (veja a Tabela 2) que identificam aqueles que geram os menores valores e, portanto, indicam os melhores ajustes. Neste trabalho foram considerados os critérios de informação de Akaike (AIC) e o Bayesiano de Schwarz (BIC), conforme são apresentados na tabela a seguir:

Tabela 2. Critérios de Informação

\begin{tabular}{|c|c|c|}
\hline Modelo & AIC & BIC \\
\hline CHEN $^{-1}$ & 795,47 & 808,58 \\
\hline LOG-NORMAL & 784,67 & 897,78 \\
\hline LOG-LOGISTICA & 783,98 & 797,09 \\
\hline
\end{tabular}

Embora os modelos Log-Normal e LogLogística apresentem os valores dos critérios de informações menores, as diferenças não são significantes entre os três valores e por isso nenhuma conclusão deve ser tomada em relação ao melhor ajuste.

Graficamente, os modelos ajustados cujos comportamentos das curvas de sobrevivência se encontram dentro da região de confiança fornecida pelo estimador de Kaplan-Meyer (Figura 4) foram considerados aceitáveis. O modelo $\mathrm{Chen}^{-1}$ se mostrou um pouco melhor no ajustamento para a região de confiança gerada devido o fato da sua curva de sobrevivência romper os limites da região de confiança durante um intervalo de tempo menor em relação aos outros dois modelos. Ou seja, aproximadamente nos intervalos de tempo $t \in[40 ; 50]$ no modelo Chen $^{-1}$, em $t \in[0,115]$ no Log-Normal e $t \in[150,350]$ no Log-Logística.

Embora a decisão em assumir que o melhor ajuste da curva de sobrevivência seja atribuído ao modelo $\mathrm{Chen}^{-1}$, tal decisão 
pode ser considerada subjetiva ao leitor através da análise gráfica, uma vez que, mesmo que a curva se encontre dentro da região de confiança durante mais tempo de duração, os três modelos rompem os limites estipulados. Além disso, os critérios de informação dos três modelos não são significativos para reforçar tal decisão.

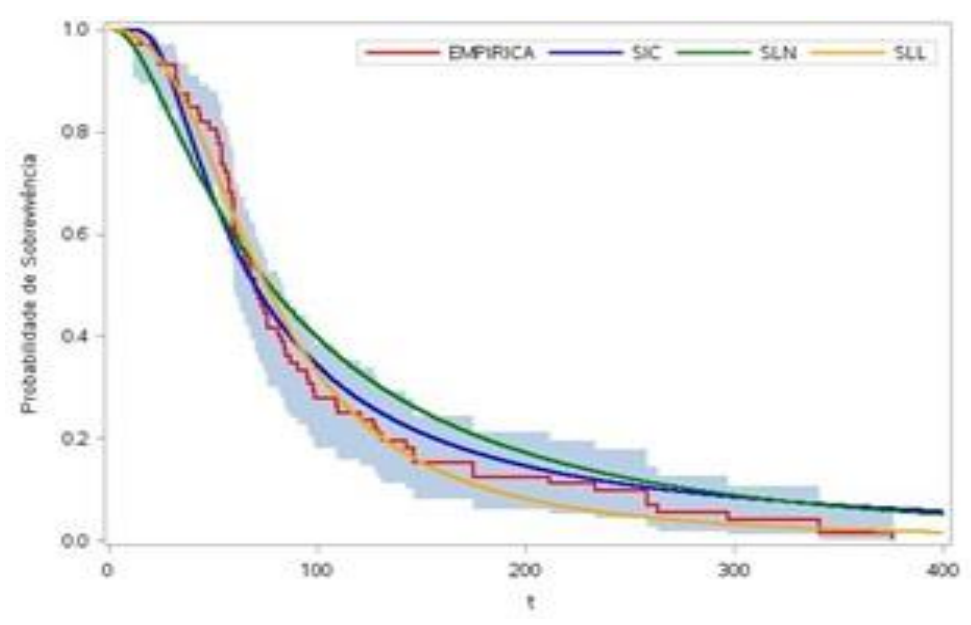

Figura 4. Gráfico de sobrevivência para as Cobaias

Contudo, uma terceira comparação se fez necessária para a conclusão do melhor ajustamento e, por este motivo, uma segunda análise gráfica foi realizada para verificar a forma da função de risco estimada pelos três modelos e comparadas com a função de risco empírica obtida dos dados do Quadro 1.
A Figura 5 apresenta as formas das funções de risco dos três modelos comparados com a estimativa da função de risco empírica, usando o método baseado em kernel proposto por Hess et al. (1999).

Na sequência é apresentado o $E Q M$ (erro quadrático médio) dos três modelos de risco em relação a taxa de falha empírica.

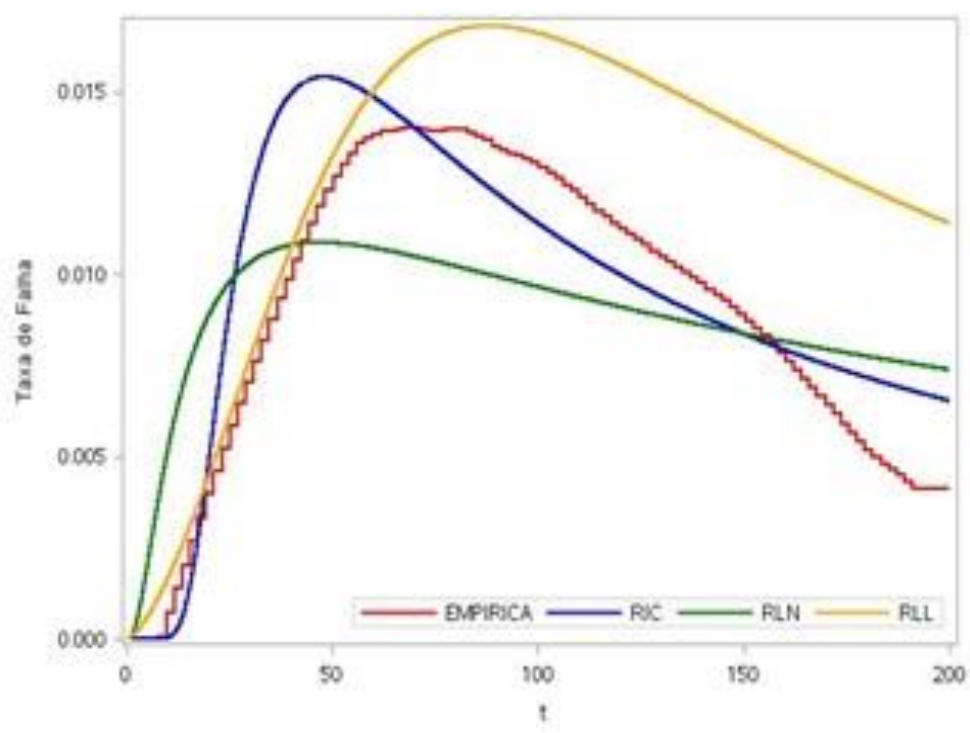

Figura 5. Curvas de risco estimadas 
Tabela 3. EQM entre os modelos de risco estimados e o empírico

\begin{tabular}{|c|c|c|c|}
\hline Modelo & CHEN $^{-1}$ & LOG-NORMAL & LOG-LOGISTICA \\
\hline EQM & $4,6 \times 10^{-6}$ & $8,0 \times 10^{-6}$ & $1,65 \times 10^{-5}$ \\
\hline
\end{tabular}

\section{CONCLUSÃO}

Portanto, os resultados das comparações gráficas e analíticas das funções de sobrevivência e de risco evidenciam o melhor desempenho do modelo $\mathrm{Chen}^{-1}$ em relação aos modelos, Log-Normal e Log-Logística, considerado os dados sobre os tempos de vida das cobaias infectadas com doses do bacilo da tuberculose.

Tal conclusão é evidenciada pelo EQM da tabela 3 , onde constata-se que $4,6 \times 10^{-6}<$ $8,0 \times 10^{-6}<1,65 \times 10^{-5}$. Essa desigualdade garante que o modelo $\mathrm{Chen}^{-1}$ apresenta o melhor ajustamento aos dados do experimento considerado.

\section{AGRADECIMENTOS}

Os autores agradecem o revisor da comissão científica pelas valiosas sugestões.

\section{REFERÊNCIAS}

BJERKEDAL, T. Acquisition of resistance in guinea pigs infected with different doses of virulent tubercle bacilli. American Jornal Epidemiology, Oxford, v.72, p.130-148, 1960.

https://doi.org/10.1093/oxfordjournals.aje.a 120129

COLOSIMO, E.A.; GIOLO, S.R. Análise de sobrevivência aplicada. 1. ed. São Paulo: Edgard Blucher, 2006. 392p.
HESS, K.R.; SERACHITOPOL, D.M.; BROWN, B.W. Hazard function estimators: a simulation study. Statistics in Medicine, v.18, p.3075-3088, 1999. https://doi.org/10.1002/(SICI)10970258(19991130)18:22<3075::AIDSIM244>3.0.CO;2-6

MORALES, M.M. Métodos alternativos à utilização de animais em pesquisa científica: mito ou realidade?. Ciência e Cultura, São Paulo, v.60, p.33-36, 2008.

SANT'ANNA, C. C.; HIJJAR, M. A. Recente contribuição da Organização Mundial de Saúde para o controle da tuberculose na infância. Revista de Saúde Pública, São Paulo, v.41, p.117-120, 2007. https://doi.org/10.1590/S003489102007000800016

SRIVASTAVA, P. K.; SRIVASTAVA R. S. Two parameter inverse chen distribution as survival model . International Journal of Statistika and Mathematika, Gorakhpur, v.11, p.12-16, 2014. 